Q. Huang ${ }^{1}$, D. Opstelten ${ }^{2}$, N. Samman ${ }^{*}$, and $\mathrm{H}$. Tideman ${ }^{1}$

${ }^{1}$ Department of Oral \& Maxillofacial Surgery, Faculty of Dentistry, Prince Philip Dental Hospital, 34 Hospital Road, and ${ }^{2}$ Department of Biochemistry, Faculty of Medicine,

The University of Hong Kong, Hong Kong;

*corresponding author, nsamman@hkucc.hku.hk

J Dent Res 81(3):209-213, 2002

\section{Experimentally Induced Unilateral Tooth Loss: Histochemical Studies of the Temporomandibular Joint}

\section{INTRODUCTION}

$\mathbf{T}$ emporomandibular joint (TMJ) osteoarthrosis is thought to result from an imbalance between predominantly chondrocyte-controlled anabolic and catabolic processes and leads to altered joint morphology due to structural changes of the tissues concerned and joint remodeling (de Bont, 1996). Although the etiology of TMJ osteoarthrosis is still not fully elucidated and is certainly multifactorial (Dijkgraaf et al., 1995; Haskin et al., 1995), occlusal abnormalities are considered as one possible factor contributing to TMJ osteoarthrosis (Kamelchuk and Major, 1995). Biomechanical factors such as occlusal and masticatory dysfunction, loss of posterior teeth, unilateral chewing patterns, and bruxism have been proposed to be involved in the initiation and progression of degenerative TMJ disease through absolute or relative overloading of joint structures (Haskin et al., 1995). Histological studies have shown that unilateral tooth extraction leads to changes in the TMJ. Studies in humans and rats show disturbance of the local microcirculation, wavy irregular fibers in the disc, smaller and condensed chondrocytes in the condylar cartilage, and destruction of articular cartilage of the TMJ (Granados, 1979; Christensen and Ziebert, 1986; Fujita and Hoshino, 1989; Ma and Pi, 1993; Hu et al, 1996). Thickening of the TMJ articular cartilage in rats after unilateral and bilateral removal of teeth (Furstman, 1965), and in Macaca mulatta with occlusal splints (Gianelly et al., 1970), has also been observed by light microscopy.

TMJ cartilage is mainly composed of water, collagen, and proteoglycans. Over $90 \%$ of the weight of the proteoglycan molecule in the extracellular matrix of normal TMJ cartilage is made up of negatively charged glycosaminoglycans (GAGs), mostly sulfated GAGs (Muir, 1983; Dijkgraaf et al., 1995). Sulfated GAGs, found in tissues normally exposed to load, may bind to cationic dyes such as safranin O. Safranin O staining is increased in the condylar cartilage of rabbits with unilateral bite raise (Mao et al., 1998). With increased mechanical force on the rat TMJ, synthesis of GAGs is increased, as shown by autoradiography (Corpray et al., 1985). Analysis of these data indicates that TMJ chondrocytes respond to changes in mechanical force, leading to an increase in GAG content of the condylar cartilage.

Since persistent unilateral mastication is likely to generate abnormal mechanical loading of the TMJ, this study poses the question whether change in the content of GAGs is part of the mechanism of TMJ cartilage degeneration in response to unilateral mastication. With the rabbit as the experimental model, an answer to this question was sought through investigation of changes following the unilateral removal of teeth. The TMJ condyle and disc were analyzed for histological parameters as well as expression pattern and levels of negatively charged ions.

\section{MATERIALS \& METHODS}

A total of 15 adult male New Zealand rabbits (18-21 wks old; 3-3.5 kg each) was used for this study. The project was approved by the Committee for the Use of Living Animals in Teaching and Research at the University of Hong Kong. After 
Table 1. Changes in Morphology of Cell Nuclei in Condylar Cartilage after the Unilateral Removal of Teeth: Number of Affected TMJs

\begin{tabular}{|c|c|c|c|c|c|c|c|c|c|c|}
\hline \multirow{3}{*}{$\begin{array}{l}\text { Morphological } \\
\text { Features } \\
\text { of Nuclei }\end{array}$} & \multicolumn{5}{|c|}{ Fibrous Layer } & \multicolumn{5}{|c|}{ Pre-chondroblast Layer } \\
\hline & \multirow{2}{*}{ Normalc } & \multicolumn{2}{|c|}{$3 w k s^{d}$} & \multicolumn{2}{|c|}{$6 w k s^{d}$} & \multirow{2}{*}{ Normal } & \multicolumn{2}{|c|}{3 wks } & \multicolumn{2}{|c|}{6 wks } \\
\hline & & Non-functional & Functional & Non-functional & Functional & & Non-functional & Functional & Non-functional & Functional \\
\hline \multirow{3}{*}{$\begin{array}{l}\text { Elongated } \\
\text { Oval/rounda } \\
\text { Irregular/ } \\
\text { condensed }^{b}\end{array}$} & 3 & 2 & & 1 & 1 & & & & & 1 \\
\hline & 3 & 1 & 1 & 4 & 3 & 6 & & 1 & 3 & 1 \\
\hline & & 3 & 5 & 1 & 2 & & 6 & 5 & 3 & 4 \\
\hline & \multicolumn{5}{|c|}{ Functional Chondroblast Layer } & \multicolumn{5}{|c|}{ Hypertrophic Chondroblast Layer } \\
\hline \multirow{2}{*}{$\begin{array}{l}\text { Oval/round } \\
\text { Irregular/ } \\
\text { condensed }\end{array}$} & 6 & & 3 & 2 & 3 & 4 & 2 & 2 & 3 & 5 \\
\hline & & 6 & 3 & 4 & 3 & 2 & 4 & 4 & 3 & 1 \\
\hline $\begin{array}{ll}\text { a } & \text { Predomin } \\
\text { b } & \text { Predomin } \\
\text { c } & \text { Two TMJs } \\
\text { d } & \text { Rabbits 3 }\end{array}$ & $\begin{array}{l}\text { nt feature } \\
\text { nt feature } \\
\text { for each n } \\
\text { or } 6 \text { wks a }\end{array}$ & $\begin{array}{l}\text { additionally, } \\
\text {; additionally, } \\
\text { ormal rabbit ( } \mathrm{r} \\
\text { after the unilate }\end{array}$ & $\begin{array}{l}\text { longated } n \\
\text { elongated a } \\
=3 \text { ) indivi } \\
\text { al removal }\end{array}$ & $\begin{array}{l}\text { ei may have be } \\
\text { /or oval/round } \\
\text { ally contribute to } \\
\text { teeth; } n=6 \text { for }\end{array}$ & $\begin{array}{l}\text { en identifie } \\
\text { nuclei may } \\
\text { o these datc } \\
\text { each time }\end{array}$ & $\begin{array}{l}\text { been id } \\
\text { I TMJs } \\
\text { total TM }\end{array}$ & $\begin{array}{l}\text { lentified. } \\
6 \text { ). } \\
\text { Us }=12 \text { for } \mathrm{e}\end{array}$ & h time & & \\
\hline
\end{tabular}

being anesthetized by intramuscular injection with a mixture of ketamine $(10 \mathrm{mg} / \mathrm{kg})$, xylazine $(5 \mathrm{mg} / \mathrm{kg})$ and acepromazine $(1$ $\mathrm{mg} / \mathrm{kg}), 12$ rabbits underwent extraction of their lower right side teeth, and were retained in the animal facility on a soft diet. They were killed by an overdose of anesthetic drug either 3 wks ( 6 rabbits) or 6 wks ( 6 rabbits) later. Three normal rabbits without extractions and living in the same conditions served as controls. The TMJs were removed en bloc and immediately fixed in $3.7 \%$ neutral buffered formalin solution. After decalcification in $10 \%$ EDTA containing dimethylsulfoxide ( $\mathrm{pH}$ 7.2) for $3 \mathrm{wks}$ and being embedded, 5 - $\mu \mathrm{m}$-thick sections were cut by means of a microtome, kept in a dry warm oven for $1 \mathrm{hr}$ at $60^{\circ} \mathrm{C}$, dewaxed, and rehydrated by serial immersion in xylene, absolute alcohol, 95\%, and 70\% alcohol, and distilled water for 5 min each.

\section{Hematoxylin \& Eosin Staining}

Sections were incubated in Harris' hematoxylin $(0.75 \%$ w/v) for 12 min and then immersed in acid alcohol for $30 \mathrm{sec}$ and in Scott's tap water for $2 \mathrm{~min}$, and stained with $1 \%$ (w/v) aqueous eosin for 5 min. The sections were washed by running tap water before and after each solution, dehydrated in serial alcohol, and mounted with Permount (BDH Limited, Poole, England). The results were photographed with a CONTAX 167MT camera attached to a Zeiss AXIOSKOP microscope (Zeiss, Germany).

On the basis of our own microscopic observations and literature review, the criteria of Engel et al. (1990) for zone delineation of rabbit condylar cartilage were used in this study. Thicknesses of condylar cartilage and disc were judged by assessment of the thickness of the central one-third region of the cartilage. Layers within condylar cartilage were classified by morphological characteristics of the nuclei (elongated, oval/round, or irregular/condensed) of the majority of cells (Table 1).

\section{Safranin O Staining}

Sections were immersed in celestine blue-alum hematoxylin solution and in Mayer's hematoxylin solution for $5 \mathrm{~min}$ each, and immersed in Scott's tap water for $1 \mathrm{~min}$. After being stained with $0.02 \%(\mathrm{w} / \mathrm{v})$ fast green for $3 \mathrm{~min}$ and rinsed in $1 \%(\mathrm{v} / \mathrm{v})$ glacial acetic acid for $30 \mathrm{sec}$, sections were stained with $0.03 \%(\mathrm{w} / \mathrm{v})$ safranin $\mathrm{O}$ for $5 \mathrm{~min}$, then dehydrated and mounted as above.
A JVC TK-C1380 color video camera was attached to a LEICA DMLB microscope, and each section was analyzed by a computerized image analyzer system (Leica Qwin, version 2.3, Leica Microsystems Imaging Solutions, Ltd., Cambridge, UK). The central one-third of the condylar cartilage was selected for quantitation. The computer program assigned an intensity to each color, red (R), green (G), and blue (B), on a scale of 0 to 255 . The proportion of red color to the whole color represented primarily safranin $\mathrm{O}$ binding and was calculated by the formula: $r=R /\left[\mathrm{R}^{2}+\right.$ $\left.\mathrm{G}^{2}+\mathrm{B}^{2}\right]^{1 / 2}$. For each TMJ, we calculated the red content of a region $\left(\mathbf{r}_{\mathbf{a}}\right.$ ) by averaging the $\mathrm{r}$ values of $3525 \mu \mathrm{m} \times 25 \mu \mathrm{m}$ squares in that region. For each group, the mean \pm SD of $6 \mathbf{r}_{\mathrm{a}}$ values was calculated. Groups were compared by the Independent Group $t$ test, with the use of SPSS statistical software. Levels of $p<0.05$ were considered significant.

\section{RESULTS}

In normal rabbits, nuclei of cells in the fibrous $(F)$ layer of the condylar cartilage were predominantly elongated, but also oval or round. In the pre-chondroblast (PC) layer, nuclei were predominantly round or oval, while a few were elongated. Compared with the PC layer, the functional chondroblast (FC) layer contained an even further increased proportion of cells with round or oval nuclei. Cells in the hypertrophic chondroblast (HC) layer were larger than in any of the other layers. Nuclei of these cells were mostly round; a few were irregular or condensed (Table 1, Fig. 1a). In the disc, the nuclei of most cells appeared round, while a few were irregular or condensed (data not shown).

After the unilateral extraction of teeth, the majority of the TMJs exhibited irregular or condensed nuclei in the F, PC, and FC layers of the condylar cartilage. In normal rabbits, such nuclear morphology was not observed. At 3 wks, the proportion of rabbits showing this abnormal nuclear feature was generally larger than at 6 wks (Table 1; compare also Figs 1b and 1c with $1 \mathrm{~d}$ and $1 \mathrm{e}$ ). Among TMJs on the functional side, the abnormality most frequently occurred in the F and PC layers. Among TMJs on the non-functional side, the abnormality was most frequent in the PC and FC layers. The HC layer, which, in the TMJs of 
normal rabbits, also exhibited irregular or condensed nuclei, showed a response to unilateral removal of teeth which was similar to but somewhat weaker than that of the other layers.

At 6 wks after the unilateral extraction of teeth, condylar cartilage appeared thicker than normal in 6 of 12 TMJs, especially the PC and FC layers. No difference in thickness was apparent at $3 \mathrm{wks}$. In the disc, no obvious difference in thickness from normal was shown in the
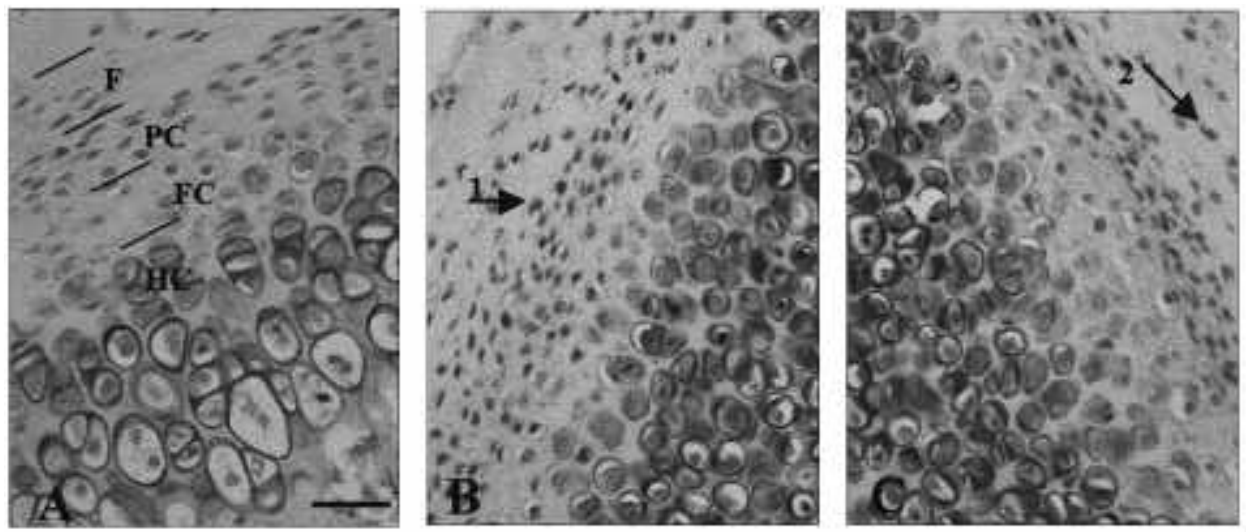
experimental rabbits.
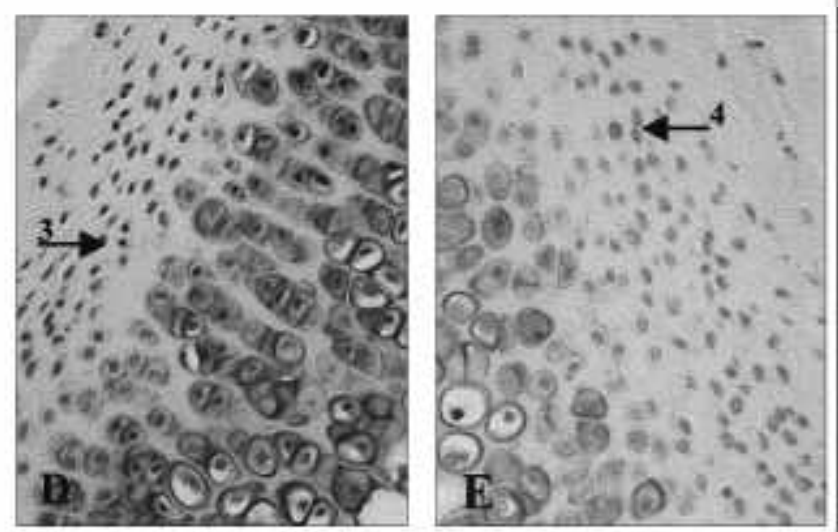
normal and experimental rabbits. However, at $3 \mathrm{wks}, \mathbf{r}_{\mathbf{a}}$ values for the FC layer of the non-functional side of TMJ, and the HC layer of both the functional and non-functional sides of TMJ, were significantly higher than normal. At $6 \mathrm{wks}, \mathbf{r}_{\mathbf{a}}$ values for the $\mathrm{HC}$ layer of the non-functional side were also significantly higher than normal. The difference in $\mathbf{r}_{\mathbf{a}}$ values for the HC layer of the functional side of TMJs between normal and experimental rabbits at 6 wks was slightly less significant. Differences in $\mathbf{r}_{\mathbf{a}}$ values between the functional and non-functional sides of TMJs were not significant at either time point.

\section{DISCUSSION}

\section{Unilateral Removal of Teeth: Changes in Condylar Cartilage and Disc of TMJ}

Thickening of condylar cartilage was identified in part of the TMJs from rabbits 6 wks following tooth extraction. In

Figure 1. Photomicrographs of sections of mandibular condyle stained with hematoxylin and eosin. (A) Normal rabbit; (B) non-functional side of TMJ 3 wks after unilateral removal of teeth; (C) functional side of TMJ 3 wks after unilateral removal of teeth; (D) non-functional side of TMJ 6 wks after unilateral removal of teeth; and (E) functional side of TMJ 6 wks after unilateral removal of teeth. F, fibrous layer; $\mathrm{PC}$, pre-chondroblast layer; FC, functional chondroblast layer; and HC, hypertrophic chondroblast layer. Arrows $1 \& 2=$ irregular nuclei; arrows $3 \& 4=$ round and oval nuclei. Bar $=40 \mu \mathrm{m}$.

addition, at 6 wks, more TMJs than at 3 wks could be classified as having predominantly oval/round nuclei in all layers of the cartilage. Both unilateral and bilateral tooth extraction in rats have been reported to cause an increase in thickness of articular cartilage of the condyle and disc (Furstman, 1965). Occlusal loss has been reported to increase loading on the articular tissues and increase the thickness of articular cartilage, as

Table 2. Changes in Safranin O Binding Intensity in Condylar Cartilage of TMJ after the Unilateral Removal of Teeth

\begin{tabular}{|c|c|c|c|c|c|}
\hline Layers of Condylar Cartilage & Normala & $\begin{array}{c}3 \mathrm{Wks}^{\mathrm{b}} \\
\text { Non-functional Side }\end{array}$ & $\begin{array}{l}3 \mathrm{Wks} \\
\text { Functional Side }\end{array}$ & $\begin{array}{c}6 \mathrm{Wks}^{\mathrm{b}} \\
\text { Non-functional Side }\end{array}$ & $\begin{array}{l}6 \mathrm{Wks} \\
\text { Functional Side }\end{array}$ \\
\hline Fibrous & $0.54 \pm 0.02^{c}$ & $0.54 \pm 0.02$ & $0.53 \pm 0.04$ & $0.55 \pm 0.01$ & $0.55 \pm 0.01$ \\
\hline Pre-chondroblast & $0.54 \pm 0.02$ & $0.54 \pm 0.02$ & $0.53 \pm 0.01$ & $0.55 \pm 0.01$ & $0.55 \pm 0.01$ \\
\hline Functional chondroblast & $0.60 \pm 0.01$ & $0.63 \pm 0.01^{*}$ & $0.62 \pm 0.02$ & $0.62 \pm 0.02$ & $0.61 \pm 0.01$ \\
\hline Hypertrophic chondroblast & $0.72 \pm 0.02$ & $0.82 \pm 0.04^{*}$ & $0.84 \pm 0.05^{*}$ & $0.82 \pm 0.06^{*}$ & $0.80 \pm 0.06^{* *}$ \\
\hline
\end{tabular}

* $\quad p<0.05$ vs. normal.

** $p=0.056$ vs. 0 normal; refer to MATERIALS \& METHODS for statistical method.

a The two sides of TMJ of normal rabbits $(n=3)$ individually contributed to these data.

b Rabbits 3 or 6 wks after the unilateral removal of teeth; $n=6$ for each time point; TMJs from the non-functional and functional sides were separately subjected to quantitation.

c $\quad r_{a}$ value, mean $\pm S D$; refer to MATERIALS \& METHODS for calculation of $r_{a}$ value. 


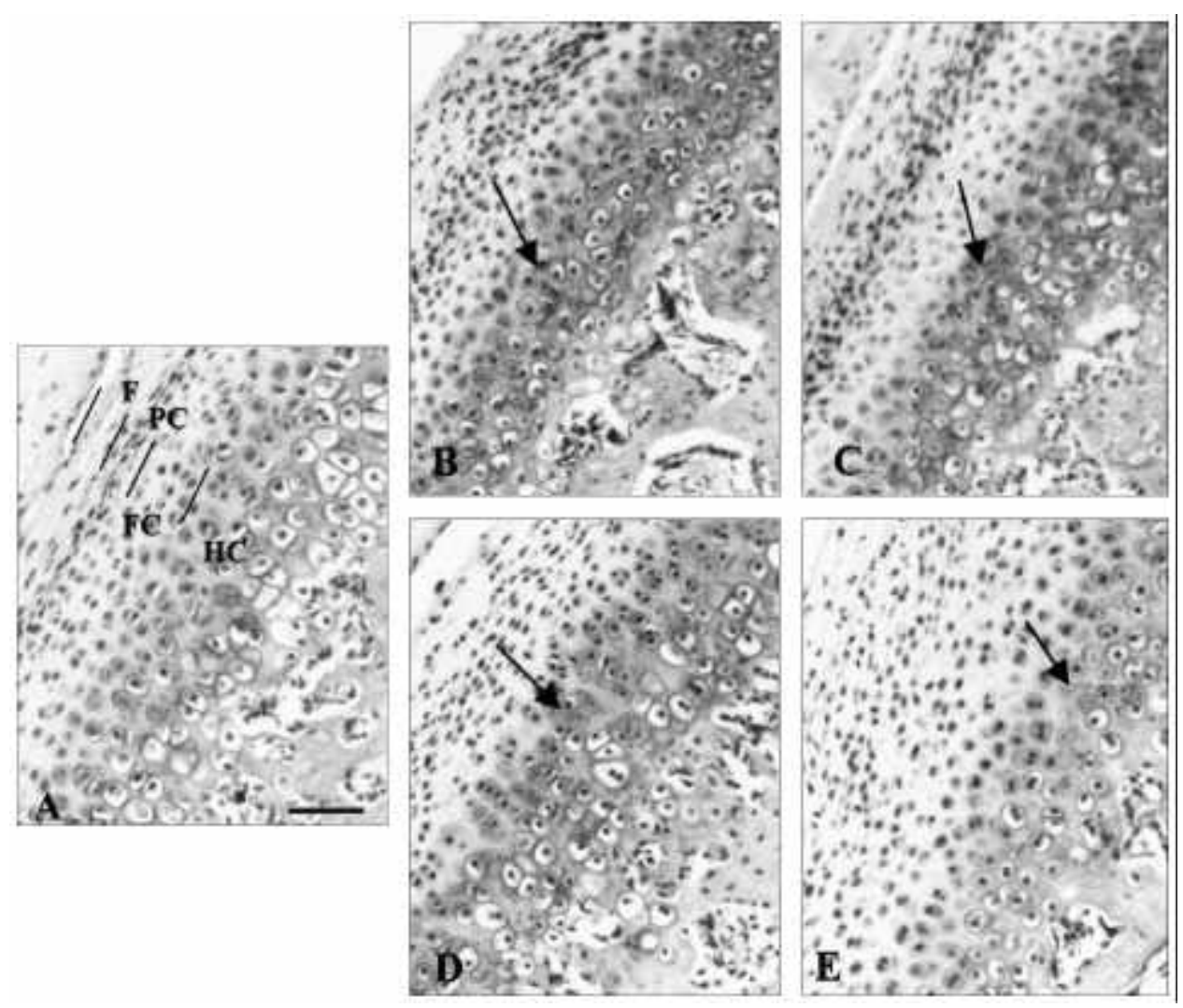

Figure 2. Photomicrographs of sections of mandibular condyle stained with safranin $O$. (A) Normal rabbit; (B) non-functional side of TMJ 3 wks after unilateral removal of teeth; (C) functional side of TMJ 3 wks after unilateral removal of teeth; (D) non-functional side of TMJ 6 wks after unilateral removal of teeth; and (E) functional side of TMJ 6 wks after unilateral removal of teeth. F, fibrous layer; PC, prechondroblast layer; FC, functional chondroblast layer; and HC, hypertrophic chondroblast layer. Arrows: safranin $O$ staining with an intensity obviously higher than normal. Bar $=80 \mu \mathrm{m}$.

shown by light microscopy (Ishimaru et al., 1994). The condylar cartilage of Macaca mulatta with occlusal splints increases in thickness (Gianelly et al., 1970). Thickening is commonly found in joints affected with osteoarthrosis (Helmy et al., 1988). The observed increase in thickness and frequency of round/oval nuclei are compatible with a remodeling response that includes increased cell proliferation and maturation.

The alteration in nuclear shape reported here suggests that, due to unilateral removal of teeth, chondrocytes in condylar cartilage and disc were metabolically disturbed in all experimental rabbits. Since no signs of cell death were observed, the presence of irregular or condensed nuclei may indicate a temporary inhibition of normal activity. The tissue appears to rebound by $6 \mathrm{wks}$, with the increase in oval/round nuclei pointing to increased metabolic activity. Other studies have reported changes in cell size. Light and scanning electron microscopy have shown that chondrocytes in condylar cartilage of the mouse with unilateral tooth amputation become smaller and condensed (Ma and $\mathrm{Pi}, 1993$ ). Enlarged spheroid fibroblasts in condylar cartilage of the rat associated with weaning, observed by electron microscopy, indicate a remodeling process (Copray and Liem, 1989). Hyperplasia of chondrocytes is reported in the proliferative and fibrocartilagenous zone of condylar cartilage of the rabbit with unilateral bite raise (Mao et al., 1998). In contrast, no significant differences have been found in TMJ cartilage between normal and experimental sheep and monkeys with unilateral tooth extraction (Ramfjord et al., 1971; Ishimaru et al.,
1994). Whether the cartilage tolerates changes in articular loading well may be due to anatomical differences that exist among different models.

In this study, an increase in the levels of negatively charged ions in condylar cartilage was positively associated with the unilateral removal of teeth. This is in agreement with Mao et al. (1998), who detected increased safranin $\mathrm{O}$ binding in the mandibular condyles of rabbits with unilateral bite raise. An increase of GAGs content was reported as positively associated with increased mechanical forces induced in the rat TMJ (Copray et $a l ., 1985)$. It is likely that an increased loading is projected onto the TMJ tissue in response to unilateral removal of teeth, causing increased expression of sulfated GAGs. This may suggest a proportional increase of GAG chains in proteoglycans, resulting in a more resistant condylar cartilage. Apparently, disc cartilage responds differently from condylar cartilage, with no measurable increase in the level of sulfated GAGs observed in this study. A study of the expression of other ECM molecules, such as chondroitin and collagen, in the TMJ cartilage would reveal additional information on changes in the molecular composition of the ECM of condylar cartilage and disc in response to unilateral tooth loss.

\section{Unilateral Removal of Teeth: Functional Side vs. Non-functional Side of TMJ}

Differences observed were in the ratio of irregular or condensed nuclei to round or oval nuclei between the functional and nonfunctional sides of TMJ after the unilateral removal of teeth. This difference in layers affected and, in "strength" of response, may be due to disproportional forces projecting onto the two sides. Biomechanical studies suggest that, due to unilateral mastication, loading of the non-functional side of the TMJ is higher than that of the functional side (Hylander and Bays, 1979). The data here on the abundance of irregular or condensed nuclei indeed indicate a stronger response in the non-functional side. However, the data are such that no statement on significance can be made.

Safranin O results showed no significant differences between the two sides. Possibly, changes in molecular composition of the ECM of TMJ cartilage were similar, even though the remodeling process following unilateral removal of teeth may not have been identical in timing and strength. Alternatively, the histochemical tool used may not be sensitive enough to detect molecular differences between two sides. Further biochemical investigation would allow for discrimination between these two alternatives. 
In conclusion, our study demonstrates that unilateral removal of teeth induces histological alterations and an increase of safranin $\mathrm{O}$ staining in TMJ condylar cartilage. Within each group of 6 experimental rabbits, the response was somewhat heterogeneous, attesting to the complexity of the mechanisms in question. The alterations observed may reflect a change in metabolic activity of chondrocytes, and a disturbance of sulfated GAGs synthesis and degradation rate, resulting in an elevated level of sulfated GAGs in the condylar cartilage of rabbits when teeth are unilaterally removed. Further studies are necessary to investigate whether the long-term effects of unilateral tooth loss in this model include TMJ osteoarthrosis.

\section{ACKNOWLEDGMENTS}

We gratefully acknowledge Mr. Yausin Ho, Ms. Anna Niewiadomska, Mr. Yingyip Chui, Dr. Guofang Shen, and Dr. Zhongchen Luo for their kind help in the experiments. This study was supported by a research grant from the Committee on Research and Conference Grants, the University of Hong Kong Grant No. 10202572.14695.08002.323.01. This paper is based in part on a thesis submitted to the Faculty of Dentistry, the University of Hong Kong, in partial fulfillment of the requirements for the Doctor of Philosophy in the discipline of Oral and Maxillofacial Surgery. Part of this study was presented at the combined 81st Annual Meeting of the American Association of Oral and Maxillofacial Surgeons and the British Association of Oral and Maxillofacial Surgeons in Boston, MA, USA, Sept., 1999.

\section{REFERENCES}

Christensen LV, Ziebert GJ (1986). Effects of experimental loss of teeth on the temporomandibular joint. J Oral Rehabil 13:587-598.

Copray JCVM, Liem RSB (1989). Ultrastructural changes associated with weaning in the mandibular condyle of the rat. Acta Anat 134:35-47.

Copray JCVM, Jansen HWB, Duterloo HS (1985). Effects of compressive forces on proliferation and matrix synthesis of mandibular condylar cartilage of the rat in vitro. Arch Oral Biol 30:299-304.

de Bont LGM (1996). Temporomandibular joint degenerative diseases: pathogenesis. In: Management of temporomandibular joint degenerative diseases. Stegenga Band de Bont LGM, editors. Switzerland: Birkhauser Verlag Basel, pp. 3-11.

Dijkgraaf LC, de Bont LGM, Boering G, Liem RSB (1995).
Normal cartilage structure, biochemistry, and metabolism. A review of the literature. J Oral Maxillofac Surg 53:924-929.

Engel FE, Khare AG, Boyan BD (1990). Phenotypic changes of rabbit mandibular condylar cartilage cells in culture. $J$ Dent Res 69:1753-1758.

Fujita S, Hoshino K (1989). Histochemical and immunohistochemical studies on the articular disk of the temporomandibular joint in rats. Acta Anat 134:26-30.

Furstman L (1965). The effect of loss of occlusion upon the mandibular joint. Am J Orthodont 51:245-261.

Gianelly AA, Ruben MP, Risinger R (1970). Effect of experimentally altered occlusal vertical dimension on temporomandibular articulation. J Prosthet Dent 24:629-635.

Granados JI (1979). The influence of the loss of teeth and attrition on the articular eminence. J Prosthet Dent 42:78-85.

Haskin CL, Milam SB, Cameron IL (1995). Pathogenesis of degenerative joint disease in the human temporomandibular joint. Crit Rev Oral Biol Med 6:248-277.

Helmy E, Bays R, Sharawy M (1988). Osteoarthrosis of the temporomandibular joint following experimental disc perforation in Macaca fascicularis. J Oral Maxillofac Surg 46:979-990.

Hu M, Zhou JL, Hong M, Wu QG, Zhang SG, Wang DS (1996). Histopathological study of miniature pig temporomandibular joint after missing teeth of one side. Chung Hua Kou Chiang Hsueh Tsa Chih 31(3):153-155.

Hylander WL, Bays R (1979). An in-vivo strain-gauge analysis of the squamosal-dentary joint reaction force during mastication and incisal biting in Macaca mulatta and Macaca fascicularis. Arch Oral Biol 24:689-697.

Ishimaru JI, Handa Y, Kurita K, Goss AN (1994). The effect of occlusal loss on normal and pathological temporomandibular joints: an animal study. J Cranio Maxillo Facial Surg 22:95-102.

Kamelchuk LS, Major PW (1995). Degenerative disease of the temporomandibular joint. J Orofac Pain 9:168-179.

Ma X, Pi X (1993). The effect of unilateral chewing on the temporomandibular joint morphology. Chung Hua Kou Chiang Hsueh Tsa Chih 28(3):151-153.

Mao JJ, Rahemtulla F, Scott PG (1998). Proteoglycan expression in the rat temporomandibular joint in response to unilateral bite raise. $J$ Dent Res 77:1520-1528.

Muir H (1983). Proteoglycans as organizers of the intercellular matrix. Biochem Soc Trans 11:613-622.

Ramfjord SP, Walden JM, Enlow RD (1971). Unilateral function and the temporomandibular joint in rhesus monkeys. Oral Surg Oral Med Oral Pathol 32:236-247. 